Nouvelles perspectives en sciences sociales

\title{
La relation comme débat fondamental de la sociologie contemporaine
}

\section{Claude Vautier}

Volume 5, numéro 1, octobre 2009

Sur le thème de la relation

URI : https://id.erudit.org/iderudit/038616ar

DOI : https://doi.org/10.7202/038616ar

Aller au sommaire du numéro

Éditeur(s)

Prise de parole

ISSN

1712-8307 (imprimé)

1918-7475 (numérique)

Découvrir la revue

Citer ce document

Vautier, C. (2009). La relation comme débat fondamental de la sociologie contemporaine. Nouvelles perspectives en sciences sociales, 5(1), 11-13.

https://doi.org/10.7202/038616ar d'utilisation que vous pouvez consulter en ligne.

https://apropos.erudit.org/fr/usagers/politique-dutilisation/ 


\section{La relation comme débat fondamental de la sociologie contemporaine}

\section{D}

ans son numéro précédent, nous avons ouvert une nouvelle rubrique dans laquelle des auteurs reconnus pour leur contribution dans un domaine particulier des sciences sociales s'expriment librement sur leur conception de ce domaine. Dans ce numéro c'est le thème de la "Relation " qui est débattu.

Cette question est centrale dans notre "Revue internationale de systémique complexe et d'études relationnelles » et, à ce titre, nous avons déjà publié des textes qui l'évoquent, au sens le plus strict dans les volume $3, \mathrm{n}^{\circ} 1$ et volume $4, \mathrm{n}^{\circ} 1$; dans un sens plus large, des articles tels que ceux de Bernard Ancori (vol. 1, $\mathrm{n}^{\circ} 1$, vol. $3, \mathrm{n}^{\circ} 2$ et vol. $4, \mathrm{n}^{\circ} 1$ ) ou de Camille Roth (vol. 2, $\mathrm{n}^{\circ} 2$ ) évoquent la théorie des réseaux sémantiques et introduisent la question du sens que nous donnons au monde que nous observons, sens créé collectivement et décidant du statut de ce que nous observons.

Il ne suffit plus aux sciences humaines contemporaines de répéter plus ou moins gauchement que l'humain est déterminé et déterminant, il faut maintenant tirer toutes les conséquences de cette importante conclusion. Et l'on ne peut le faire qu'en faisant de l'humain lui-même le lieu de la dialectique,

écrivait Simon Laflamme en $1995^{1}$.

Simon Laflamme, Communication et émotion. Essai de microsociologie relationnelle, Paris, L'Harmattan, coll. "Logiques sociales », 1995, p. 143. 
Les auteurs qui nous ont offert un texte d'opinion pour cette nouvelle livraison de Nouvelles Perspectives en Sciences Sociales essaient, chacun à sa manière, de résoudre le problème évoqué par Laflamme et par tous ceux (Donati, Emirbayer, Bajoit, Degenne, Forsé et bien d'autres) qui retrouvent et ré-explorent l'idée ancienne selon laquelle parler de "phénomènes sociaux " impose de considérer " que ces phénomènes sont situés, non dans les éléments, mais dans le tout formé par leur union " ${ }^{2}$.

On se souviendra que Weber, dans le chapitre premier de son Economie et société 1, qui traite des concepts fondamentaux de la sociologie, définit des " activités significatives" en les rapportant au sens que leur donnent les sujets humains et des " comportements réactionnels" " non associés à un sens visé subjectivement " et conclut sur ce point qu' " une part très considérable de l'ensemble des comportements sociologiques [...] se situe aux limites des deux ${ }^{3}$. L’ensemble du $\$ 1$ de ce chapitre tourne autour de l'idée que si l'on ne peut comprendre une activité significative que "sous la forme d'un comportement d'une ou plusieurs personnes singulières", "pour la sociologie [...] c'est précisément l'ensemble significatif de l'activité qui constitue l'objet de l'appréhension " ${ }^{4}$. Hésitation, dialectique entre les valeurs compréhensives du tout et de la partie? Simmel écrivant dans son essai "Pont et porte " que " nous sommes à chaque instant ceux qui séparent le relié ou qui relient le séparé " ${ }^{5}$ vient en écho de cette interrogation historique de la discipline sociologique et, plus largement des sciences sociales : quel est le statut de l'être humain dans ce que nous nommons "société "? Quel est le statut de cette "société " dans la vie des êtres humains?

Au-delà de l'idée aujourd'hui triviale que les humains font la société qui les fait, une méthode sociologique ou des sciences

$2 \quad$ Émile Durkheim, Les règles de la méthode sociologique, Préface à la première édition, Paris, PUF, coll. "Quadrige », [1937] 1999, p. XVI.

3 Max Weber, Économie et société, 1. Les catégories de la sociologie, Paris, Plon, coll. « Pocket », 1995, p. 29.

4 Ibid., p. 40. C'est nous qui soulignons les termes «singulières » et " ensemble».

5 Georg Simmel, La tragédie de la culture et autres essais, Paris, Rivages poche, 1988, p. 162. 
humaines qui tente d'approcher les phénomènes sociétaux en échappant au holisme méthodologique exclusif prêté - à tort - à Durkheim et à l'individualisme méthodologique exclusif, dont on ne pourrait qu'à tort également affubler Weber, doit résoudre les problèmes du statut respectif de la partie et du tout, du sens, de l'intentionnalité et du réactionnel, de l'activité psychique des hommes à qui seuls, selon Simmel, "il est donné face à la nature de lier et délier " ${ }^{6} .$.

S'y essayant, nos auteurs labourent le champ des possibles, ce faisant apportent des clartés contrastées sur cet objet si familier et si étrange cependant que constitue la « relation » dans les sciences sociales.

Le débat, évidemment, ne s'épuise pas avec ces contributions, malgré leurs grandes qualités. Il roule ses arguments comme un torrent ses galets espérant s'assagir demain dans un statut de question relativement consensuelle, tandis que d'autres débats ou d'autres branches de celui-ci nous redonneront, avec les polémiques, de la vigueur et de la vie.

Claude Vautier

$6 \quad$ Ibid., p. 161. 